Original

\title{
Colpopéxia transcoccígea con malla de polipropileno con orificios facilitadores de la integración en la corrección del prolapso de la pared posterior de la vagina: resultados anatómicos y funcionales
}

\author{
Vitor Pagotto, Paulo Palma, Cassio Riccetto, Miguel Bigozzi* \\ Servicio de Urología de la Facultad de Ciencias Médicas de la Universidad Estatal de Campinas. Brasil. \\ *Servicio de Ginecología, Hospital Santojanni, Buenos Aires, Argentina.
}

\begin{abstract}
Resumen
Objetivo: La creación de neoligamentos útero- sacros, según lo descripto por Petros, con el uso de malla sintética de polipropileno permite la corrección anatómica del prolapso posterior en los tres niveles propuestos por DeLancey. El objetivo de este estudio es evaluar los resultados anatómicos y funcionales de este método en la corrección del POP posterior.

Pacientes y método: Desde diciembre del 2004 a marzo del 2007, 34 mujeres con edad media de 63 años fueron sometidas al procedimiento, siendo rectoceles grado III o mayores. El seguimento mínimo fué de 13 meses. Los puntos de fijación fueron el músculo íleococcígeo y el ligamento sacroespinoso.

Técnica: Luego de la incisión de la mucosa de la pared vaginal posterior, la fosa ísquio-rectal es disecada. Dos pequeñas incisiones son hechas $3 \mathrm{~cm}$ lateral y $3 \mathrm{~cm}$ inferior al ano. La aguja se inserta verticalmente en dirección a la espina isquiática, guiado por el dedo indicador del cirujano hasta el ligamento sacroespinoso, $2 \mathrm{~cm}$ medialmente, evitando a lesión del canal de Alcock. Los brazos de la malla son conectados a la punta de la aguja, traccionando posteriormente de las agujas. No se realiza la correción sitio específica. El exceso de malla es recortado y esta se fija próxima al cuerpo perineal, quedando de esa forma extendida sobre la pared vaginal posterior. La mucosa vaginal se cierra en forma habitual.

Resultados: Con el 94,7\% de cura y recurrencia del 5,3\%, no hubo lesión nerviosa, visceral o vascular. Cinco (14,7\%) pacientes tuvieron exposición de la malla de menos de $1 \mathrm{~cm}^{2}$ y fueron tratadas conservadoramente sin impacto en los resultados. Dos de las 17 sexualmente activas $(11,8 \%)$ presentaron dispareunia transitoria, siendo prolongada en 1 solo caso $(5,9 \%)$
\end{abstract}

Conclusión: Este procedimiento mínimamente invasivo es una alternativa atractiva para la reconstrucción anatómica y funcional de los defectos posteriores y apicales.

Palabras claves: Prolapso urogenital. Retocele. Polipropileno. Colpopexia.

\section{Transcoccigeal colpopexy with polyprolylene mesh with helper orifices for the treatment of posterior vaginal wall prolapse: anatomical and functional results.}

\section{Abstract}

Objective: The creation of neo utero-sacral neoligaments, decribed by Petros, evolved into a new procedure allowing for the anatomical reconstruction of the three levels proposed by DeLancey. The aim of this study is evaluate the anatomical and functional outcomes of this minimally invasive procedure.

Patients and Method: From December 2004 to March 2007, a total of 34 patients with posterior defect grade III or higher underwent this procedure. The minimum follow up was 13 months Mean age was 63 years. The site of fixation was the Sacrospinous ligament.

Surgical Technique: The ischiorectal fossa is dissected. Next two small skin incisions are made $3 \mathrm{~cm}$ lateral and inferior to the center of the anus. A proper needle is introduced, vertically towards the sacrospinal ligament at the level of the ischial spine, guided by the surgeon index finger, $2 \mathrm{~cm}$ medially avoiding the Alcok canal. The armpit of the mesh is connected to the tip of the needle and brought to the perineal region. No site specific correction is made.

Results: The cure rate was $94,7 \%$ and recurrence rate was $5,3 \%$. No visceral, nerurovascular injuries were observed. The mesh exposure rate (less than $1 \mathrm{~cm}^{2}$ ) was $(14.7 \%)$ and all patients were treated conservatively with no impact on the outcome. There were transient dyspareunia in $2(11.8 \%)$ of the 17 sexually active patient and persistent in 1 case (5.9\%).

Conclusions: This procedure is an attractive minimally invasive alternative for the anatomical and functional reconstruction of the posterior and apical defects.

Keywords: Urogenital prolapse. Rectocele. Polypropylene.Colpopexy. 
$\mathrm{E}_{\mathrm{n}}^{\mathrm{l}}$ prolapso de los órganos pélvicos (POP) en las mujeres resulta de la protrusión de la pared anterior y posterior de la vagina, pudiendo asociarse al descenso del cuello uterino, a través del canal vaginal, con la posible exteriorización a través del vestíbulo vaginal. El aumento de la expectativa de vida de la población mundial registrada en los últimos decenios ha determinado el aumento gradual en la prevalencia de prolapsos ${ }^{1}$. Existen pocos estudios sobre su prevalencia, debido a la inconsistencia de los sintomas y los problemas de la normalización del examen vaginal ${ }^{2}$. Por lo tanto, los estudios de incidencia se limitan a los destinados a la investigación de nuevos tratamientos quirúrgicos. Olsen et al (1997) ${ }^{3}$ demostró que el riesgo que presenta una mujer de 80 años que se le practique una cirugía para corregir POP o la incontinencia urinaria es del $11,1 \%$.

Sintomas tales como sensación de "masa o bulto" vaginal, trastornos defecatorios, o trastornos sexuales pueden estar presentes en pacientes con prolapso de la pared posterior de la vagina, sin embargo, no se correlaciona, necesariamente, con la gravedad del prolapso ${ }^{4}$.

Las principales técnicas propuestas para el tratamiento del prolapso vaginal posterior son: la miorrafia de los elevadores del ano y la reconstrucción del anillo pericervical. Estas técnicas se combinan a veces con otras, para el tratamiento del prolapso uterino o de la cúpula vaginal después de la histerectomía. La elevada tasa de fracaso con esas técnicas puede explicarse en parte por la mala calidad de los tejidos, especialmente el tabique rectovaginal, los ligamentos cardinales y los ligamentos sacrouterinos.

El uso de mallas sintéticas surgió en la última década como una alternativa para el tratamiento de prolapsos de alto grado, asociada a la mala calidad de los tejidos y las recaídas ${ }^{5}$. El objetivo del estudio fue evaluar la seguridad, los resultados y las complicaciones de la colpopexia transcoccígea con malla sintética de polipropileno monofilamento para el tratamiento del prolapso de la pared vaginal posterior evaluando el impacto del tratamiento sobre la calidad de vida y la función sexual de las pacientes.

\section{PACIENTES Y MÉTODOS}

Entre diciembre de 2004 y marzo de 2007, 34 pacientes con una edad media de 63 años fueron sometidas a corrección de prolapso de la pared vagi- nal posterior con el uso de una malla sintética de polipropileno monofilamento con agujeros facilitadores de la integración (NAZCA R-POP sistema de reparación, Promedom, Argentina). Se incluyeron pacientes con prolapso de la pared vaginal posterior estadio III y IV, pacientes con Prolapsos recidivados estadio II (que corresponde a un punto Bp mayor o igual a uno, de acuerdo a cuantificación de prolapso de órganos pélvicos sistema de - POP-Q). Se excluyeron los pacientes sometidos con anterioridad a la corrección de prolapso vaginal posterior con otras mallas, inmunosuprimidos, o con infección urinaria.

La prótesis NAZCA R está confeccionada con una malla de polipropileno monofilamento tipo I, con una densidad de $60,5 \mathrm{~g} / \mathrm{m}^{2}$. Sus poros son de $0,5 \mathrm{a}$ $1 \mathrm{~mm}$ de longitud, el grosor de $0,47 \mathrm{~mm}$ y los filamentos son de 0,14 mm de diámetro. En la parte central, presenta orificios circulares con seis milímetros de diámetro, a fin de facilitar su integración con los tejidos, y también disminuir la cantidad de material sintético implantado y aumentar su flexibilidad. La malla tiene dos brazos con extremidades de silicona, para conectar a las aguja introductoras. Dos agujas con cabos removibles conforman el juego completo disponible para el procedimiento (Fig. 1a).

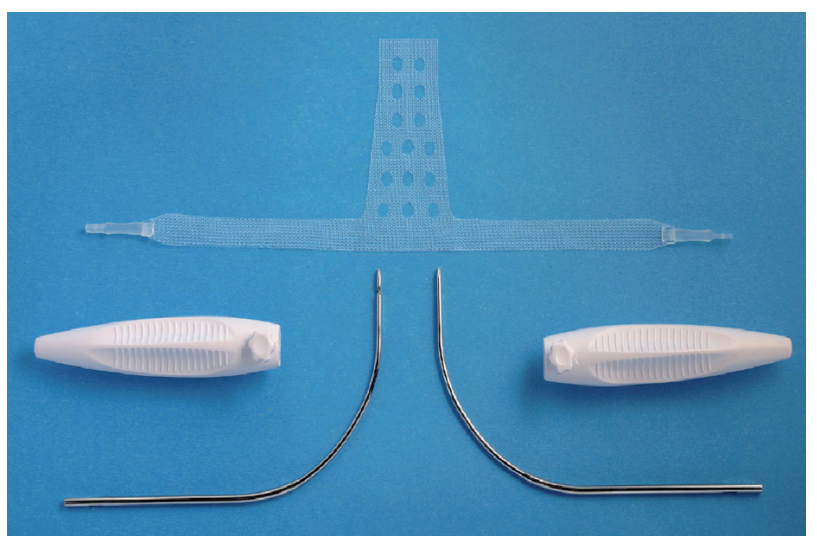

FIGURA 1a: Set de Prótesis de polipropileno monofilamento con agujas.

\section{TÉCNICA QUIRÚRGICA}

La colpopexia transcoccígea tiene por objetivo restablecer la anatomía pericervical posterior, en pacientes con compromiso anatómico y funcional del complejo útero-sacro y ligamento cardinal, así como reforzar o sustituir el tabique rectovaginal. El procedimiento se realiza con la paciente en posición 
de litotomía bajo anestesia raquídea. Realizada una incisión vaginal media, que se extiende desde la transición de la piel y la mucosa vaginal posterior hasta el fondo de saco vaginal posterior. Luego se realiza disección de la pared vaginal posterior, se identifica el septum rectovaginal y su defecto correspondiente. El recto está completamente aislado de la pared vaginal, identificándose los ligamentos sacroespinosos a cada lado. Se realizan posteriormente dos incisiones en la piel, tres centímetros por fuera y tres centímetros por debajo del ano, de forma bilateral (Fig. 1b).

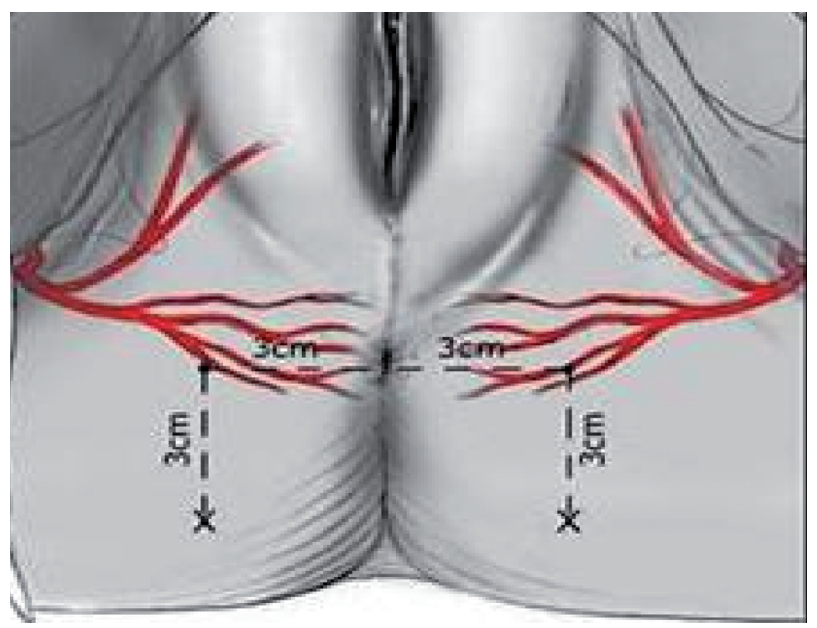

FIGURA 1b: Sitio de punción (Figura Modificada Cleveland Clinic).

La aguja se introduce por esta incisión perpendicular a la piel pasando por debajo del músculo elevador del ano, guiado por el dedo índice del cirujano desde el interior de la vagina, luego se realiza la transfixión del ligamento sacroespinoso a $2 \mathrm{~cm}$ medial de la espina isquiática (Fig. 2a). Esto se realiza para evitar la lesión del paquete vasculo-nervioso-pudendo, que se sitúa lateral a la espina isquiática. A continuación, cada hasta se conecta a la punta de la aguja, retirándose a través del sitio de punción perineal, la prótesis se ancla en el ligamento sacrospinoso ipsilateral. La malla se sutura a los ligamentos útero-sacros con puntos irreabsorbibles (Fig. 2b). Las astas son traccionadas restableciendo el apoyo apical de la vagina. (Fig. 3a) El exceso de cuerpo de la malla se corta y la longitud de la malla se ajusta a la longitud vaginal. Se procede a la sutura de la pared vaginal y la tracción adicional de las astas de fijación buscando que la cúpula vaginal se encuentra al nivel de las espinas isquiáticas, lo que

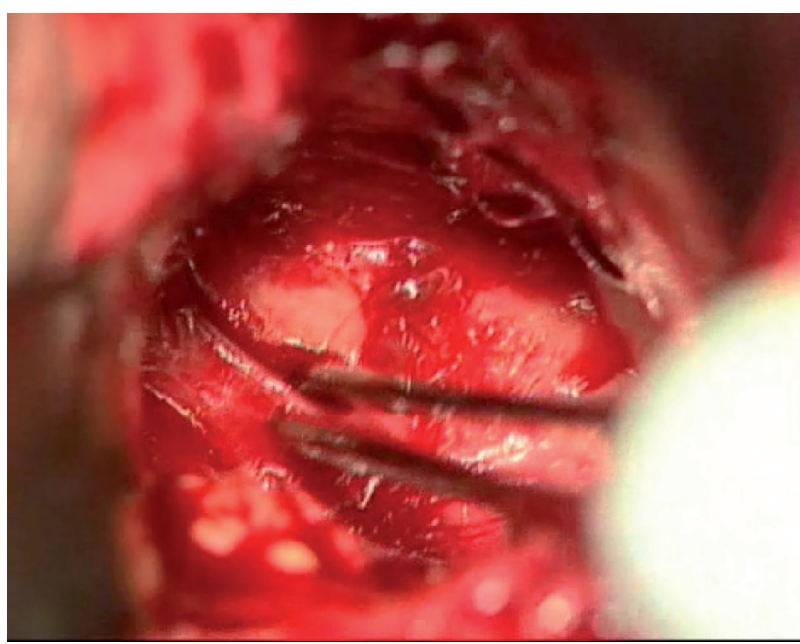

FIGURA 2a: Identificación del Ligamento Sacroespinoso.

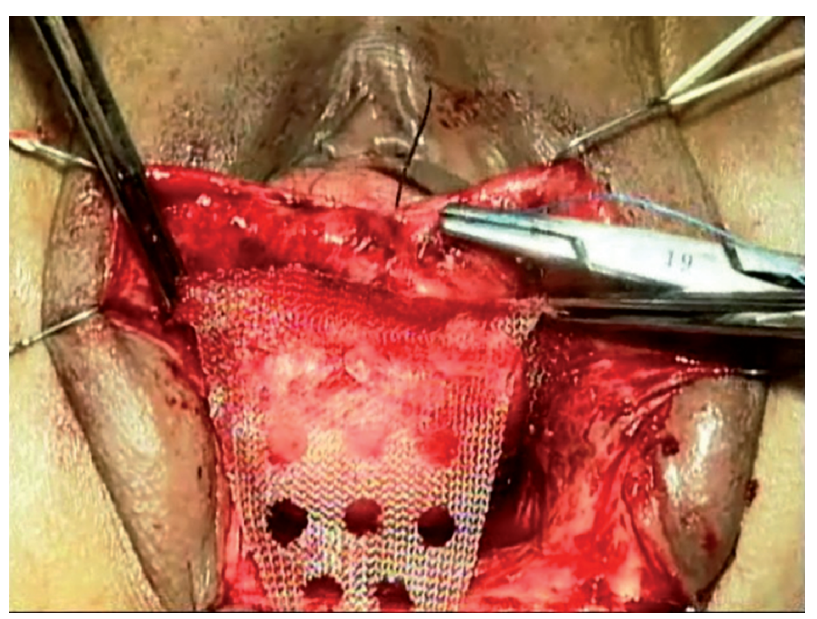

FIGURA 2b: Fijación de la malla con hilo irreabsorvible.

corresponde a su posición fisiológica. (Fig. 3b) Otros prolapsos asociados se corrigieron simultáneamente durante el procedimiento, de acuerdo con la indicación específica.

Todas las pacientes recibieron profilaxis antibiótica durante la cirugía con cefalosporinas de segunda generación en una sola dosis. La sonda vesical fue retirada después de 24 horas del procedimiento en el momento del alta hospitalaria. Durante el periodo postoperatorio, las pacientes fueron dirigidas a permanecer en reposo durante cuatro semanas. El retorno a la actividad sexual se recomienda después de 6 semanas.

Todas las pacientes fueron examinadas por el mismo médico pre y postoperatorio, uno, tres, seis, nueve y doce meses después de la cirugía, incluida la determinación del grado de prolapso a través del POP-Q. Fueron consideradas curadas las pacientes 


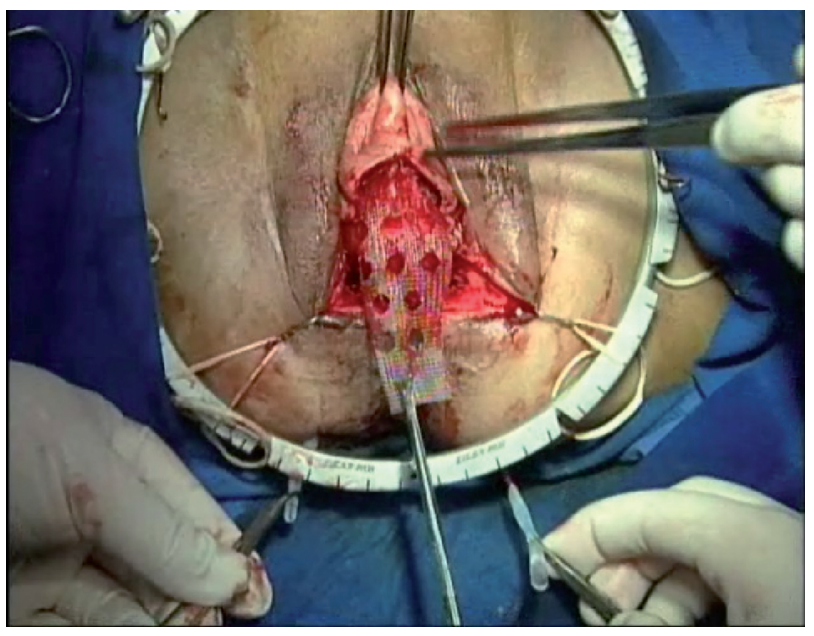

FIGURA 3a: Tracción de lo brazos.

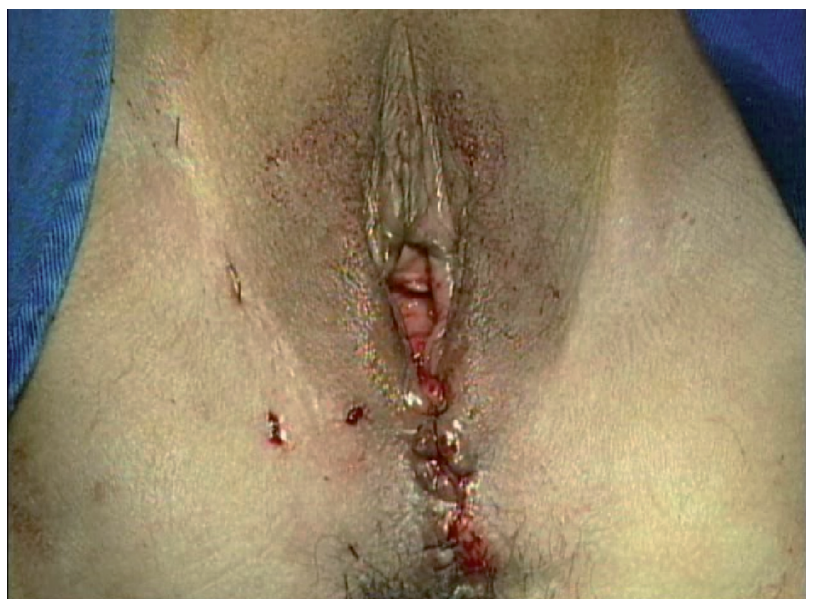

FIGURA 3b: Aspecto final del prolapso corregido.

asintomáticas y con grado de prolapso de cero o uno, es decir, con punto Bp igual o inferior a -2 después de 12 meses.

Todas las pacientes respondieron al cuestionario de Función Sexual Femenina Index (IFSF), validada para el portugués en el pre-operatorio, y después de 3 meses de seguimiento. El IFSF (índice de la función sexual femenina) instrumento multidimensional, breve que mide la función sexual femenina (Rosen et al 2000), consta de 19 preguntas que informan sobre 5 dominios de respuesta sexual: deseo o estímulo subjetivo, lubricación, orgasmo, placer y dolor o discomfort. Puntuaciones individuales se obtienen sumando los ítems que componen cada dominio (puntuación única), que se multiplica por el factor de ese dominio, determinando una puntuación ponderada. La puntuación final (puntuación total: mínimo 2 y máximo de 36 ) se obtiene mediante la suma ponderada de las puntuaciones de cada dominio.

\section{RESULTADOS}

Fueron estudiados un total de 34 pacientes, 24 $(70,5 \%)$ tenían prolapso posterior estadio III y 10 pacientes $(29,5 \%)$ estadio IV, de acuerdo con el POPQ. El promedio pre-operatorio de $\mathrm{Bp}$ fue de $+3 \mathrm{y}$ el punto D igual a -3 . Siete pacientes $(20,5 \%)$ tenían sintomas de estreñimiento y necesidad de asistencia defecatoria manual y 3 pacientes tenían prolapso de cúpula vaginal asociado con prolapso de pared posterior. En diez pacientes, el prolapso de la pared vaginal anterior se corrigió simultáneamente, y en cinco pacientes el tratamiento de la incontinencia urinaria de esfuerzo también fue realizado. Ninguna paciente presentó prolapso rectal.

La edad media fue de 63 años, variando entre 50 a 85 años. Todas se encontraban en menopausia y ninguna de ellas recibió terapia de reemplazo hormonal. La media de embarazos fue de 5,2, y la media de partos normales fue de 4,2. El indice de masa corporal medio fue de 27,7. Diez pacientes habian presentado anteriormente corrección de prolapso de la pared vaginal anterior y posterior, sin la utilización de malla.

La duración media de hospitalización fue de 30 horas y el tiempo mínimo de seguimiento fue de 13 meses. No se observaron complicaciones durante la cirugía y el sangrado fue insignificante, por lo que ninguna de las pacientes recibió transfusiones de sangre. Dos pacientes tuvieron infección del tracto urinario durante el postoperatorio inmediato. No se registraron infecciones de la prótesis o fístula rectovaginal durante el seguimiento.

Treinta y tres pacientes $(97 \%)$ realizaron seguimiento ambulatorio, con determinación de POP-Q, siempre por el mismo médico. Durante el seguimiento el punto Bp tuvo una media de $-2,2$ y el punto D medio de $-5,1$ después de 12 meses, demostrando ser significativamente diferente con respecto a la evaluación preoperatoria $(\mathrm{p}<0,0001)$. Después de 12 meses de seguimiento, el 42,1\% de las pacientes se encontraban en estadio 0 de prolapso de la pared vaginal posterior y $52,6 \%$ en estadio I (Fig. 1).

Sin embargo, se observó que el 5,3\% estaban en estadio II, considerando fracaso del procedimiento. Por lo tanto el 94,7\% de las pacientes fueron considerados curadas (Tabla 1).

Cinco pacientes $(14,7 \%)$ tuvieron exposición vaginal de la malla (todas inferiores a $1 \mathrm{~cm}$ de diámetro). En todos los casos, la exposición se encon- 
Tabla 1. Mediana de los puntos pre y pos operatorio

\begin{tabular}{lccccccc}
\hline Medida & Inicial & $\mathbf{1}$ mes & $\mathbf{3}$ meses & $\mathbf{6}$ meses & $\mathbf{9}$ meses & $\mathbf{1 2}$ meses & pos 12 meses \\
\hline Aa & $-2,0$ & $-2,0$ & $-2,0$ & $-2,0$ & $-1,5$ & $-1,5$ & 0,2680 \\
Ba & $-1,0$ & $-2,0$ & $-2,0$ & $-1,0$ & $-1,0$ & $-1,0$ & 0,1061 \\
C & $-6,0$ & $-6,0$ & $-6,0$ & $-5,5$ & $-5,5$ & $-5,5$ & 0,2852 \\
Ap & 2,0 & $-3,0$ & $-3,0$ & $-3,0$ & $-3,0$ & $-3,0$ & $<0,0001$ \\
Bp & 3,0 & $-3,0$ & $-3,0$ & $-3,0$ & $-3,0$ & $-3,0$ & $<0,0001$ \\
D & $-4,0$ & $-6,0$ & $-6,0$ & $-6,0$ & $-6,0$ & $-6,0$ & $<0,0001$ \\
Gh & 4,0 & 4,0 & 4,0 & 4,0 & 4,0 & 4,0 & $* *$ \\
Pb & 3,0 & 3,0 & 3,0 & 3,0 & 3,0 & 3,0 & $<, 0$ \\
TVL & 8,0 & 8,0 & 8,0 & 8,0 & 8,0 & $*, 0001$ & $* *$
\end{tabular}

tró en la porción distal de la malla, al lado del vestíbulo vaginal en la línea de sutura. Tres pacientes informaron presencia de flujo vaginal, una paciente informó que la pareja se quejó de dolor durante el coito, en la otra, la exposición fue asintomática. Cuatro de ellas fueron sometidas a resección parcial de la malla y la sutura de la pared vaginal bajo anestesia espinal, con buena evolución. La paciente asintomática fue tratada con estrógenos tópicos con la resolución de cuadro.

Diecisiete pacientes no eran sexualmente activas antes de la cirugía. Entre las restantes diecisiete , 2 (11,8\%) desarrollaron dispareunia después de 3 meses del procedimiento, que fue relacionado con la exposición de la malla. En ambos hubo resolución de los sintomas tras la resección de parte de la malla.

\section{DISCUSIÓN}

La sustentación de la vagina y los órganos pélvicos es el resultado de la integridad de los músculos del piso pélvico, sus nervios, las fascias y los ligamentos, representado principalmente por la fascia endopélvica. El tono muscular basal del músculo elevador del ano cierra la brecha urogenital, comprimiendo la uretra media, la vagina y el recto contra de pubis, ofreciendo resistencia al aumento de la presión abdominal. Cuando se produce daño muscular, el apoyo es ahora principalmente por el tejido fascial y ligamentario, que, después de la tensión continua, se distiende y puede sufrir rupturas, lo que lleva a la producción de prolapsos.

De las tres vías de acceso para la corrección de prolapso posterior, la vía abdominal está indicada cuando existe algún grado de prolapso rectal aso- ciado o intusucepción. El acceso transanal es adecuado para pequeños rectocele asociado con prolapso de mucosa rectal. En tales casos, la operación de Delorme (mucosectomía y plicatura múscular) se puede realizar ${ }^{7}$. Entre las críticas de esta técnica, se hace hincapié en que la dilatación puede poner en peligro al esfínter anal, con el riesgo de empeoramiento de su función, aunque en un reciente estudio con 45 pacientes, el seguimiento promedio fue de 24 meses se han registrado buenos resultados funcionales en el $77,8 \%$ de los casos sin aumentar la incidencia de la incontinencia fecal ${ }^{8}$. A pesar de la utilización de grampeadoras lineales simplificando la mucosectomía, complicaciones graves, como hemorragia, dolor anal, estrechamiento rectal y fístula rectovaginal se han descrito ${ }^{9}$.

Existen variaciones significativas en la literatura sobre las técnicas de reparación transvaginal. La plicatura transversal del músculo elevador del ano en la línea media es aceptable, con un resultado anatómico entre el 76 y el 96\%, pero con hasta un $50 \%$ de dispareunia "de novo" ${ }^{10}$. De manera contraria, el concepto de que el rectocele es consecuencia de un defecto en el tabique rectovaginal aislado, ya había sido propuesto anteriormente, así como la necesidad de su investigación sistemática durante la cirugía, condicionando el diagnostico preciso y el éxito del tratamiento quirúrgico ${ }^{11}$. Del mismo modo, se informó de la frecuencia de cada defecto vaginal especifico en un estudio retrospectivo con 106 pacientes (Burrows et al 2003), es decir, superior (27\%), inferior (27\%), izquierdo (30\%), derecho (7\%), Central (7\%) y no identificados (2\%). En el mismo estudio, se verificó que la corrección del defecto 
específico de tabique rectovaginal determinó resultados anatómicos buenos y no mostró efectos adversos sobre la función sexual, pero sólo 35-50\% de los pacientes con síntomas intestinales mostraron mejoría en sus sintomas ${ }^{12,13}$.

Hay pocos estudios comparativos relacionados con el tratamiento del prolapso vaginal posterior. En un estudio con 124 mujeres en el que se comparó la plicatura del tabique rectovaginal en la línea media con la corrección sitio-especifica para el tratamiento de los prolapsos posteriores se observó una tasa más alta de recurrencia del prolapso de la pared posterior en el grupo en el que los defectos fueron corregidos aisladamente (44\%), en comparación con el $18 \%$ en el grupo sometido a plicatura mediana sistemática del tabique rectovaginal ${ }^{12}$.

Se entiende que la elevada tasa de fracaso en la reparación puede ser el resultado de tejidos de mala calidad fascial del tabique rectovaginal. Este aspecto ha justificado la propuesta de utilizar mallas. En un estudio se analizaron prospectivamente 26 mujeres sometidas a corrección de prolapso vaginal posterior con malla de polipropileno, por 23 meses de seguimiento medio. La tasa de curación fue del $92 \%$ y el éxito funcional obtenido fue del 88\%. Sólo tres $(11,5 \%)$ pacientes tuvieron exposición vaginal de la malla y una $(3,8 \%)$ paciente desarrolló dispareunia ${ }^{14}$. En otro estudio observacional, en 90 pacientes que se sometieron a reparación del rectocele con malla de polipropileno, se llegó a la conclusión de que la presencia de la malla no ha dado lugar a dificultades técnicas, en comparación con la tradicional colporráfia. La baja incidencia de complicaciones se observó como un factor alentador para la aplicación de la técnica. Del mismo modo, en esta casuística, no se encontró hemorragia significativa durante la cirugía o formación de hematomas en el postoperatorio. También el dolor de los pacientes en el postoperatorio no fue significativo o persistente. En el mismo estudio, la incidencia de dispareunia de novo fue del 3,4\% y la tasa de exposición vaginal de la malla fue de $12,9 \%{ }^{15}$. En este estudio, la tasa de exposición vaginal de la malla fue similar. Además, en esta casuística, sólo aquellas pacientes que habían desarrollado la exposición de la malla se quejaron de dispareunia cuando se examinó a través de ISFS. Esto permite inferir que siempre que se tenga cuidado de reducir al mínimo los defectos de la integración, el riesgo de disfunción sexual será pequeño.

La revisión Cochrane del 2007 relató la superioridad de la vía vaginal sobre la transanal en cuanto al riesgo de falla subjetiva y objetiva. También dice que no hay diferencia entre la corrección del rectocele con y sin malla de poliglactina absorbible. Sin embargo, cuando comparamos la reparación sitio específica solo o con malla biológica, no se estableció beneficio claro para el empleo de la malla ${ }^{16}$.

En los primeros intentos de restablecer el apoyo vaginal, a través de las mallas sintéticas por el acceso transglúteo ${ }^{17}$ propone que el apoyo de la malla se realiza a través de la transfixión del haz îleococcígeo del elevador del ano y su fascia. El bajo riesgo de lesiones vasculares y de lesión del nervio pudendo interno con la buena descripción de resultados preliminares ha derminado que este procedimiento haya tomado repercusión $^{17}$. Según la técnica de colpopéxia infracoccígea descripta por Inmon $^{18}$, este procedimiento estipula restaurar la vagina a un nivel 2 de De Lancey ${ }^{18,19}$. Este hecho tenía una desventaja teórico potencial en comparación con técnicas como la sacropromontopexia y colpopéxia a través de la fijación sacrospinosa, con el que sería posible restablecer el ápice vaginal hasta una posición más craneal. Cabe recordar, también, que el exceso de disección (generalmente digital) de la fosa isquiorrectal podría aumentar el defecto en el tabique rectovaginal y empeorar las condiciones locales tisulares, donde la prótesis se fija, predisponiendo a la reaparición del prolapso.

La técnica de colpopéxia transcoccígea con malla utilizada en este estudio intenta reconstruir el soporte vaginal en el nivel 1 de apoyo descripto por De Lancey ${ }^{19}$, que se encuentra a nivel de las espinas isquiáticas, restaurando la porción posterior del anillo pericervical, representado por los ligamentos Sacrouterinos. También permite, de manera asociada con la corrección de rectocele, el restablecimiento de la función de la fascia rectovaginal. Por lo tanto, esta técnica modifica poco el eje vaginal normal. En este estudio, el uso sistemático de la colpopéxia transcoccígea resultó en la cura del 94,7\%, de las pacientes evaluadas objetivamente a través del POP-Q. Cabe recordar que este resultado se sobrepone a los resultados obtenidos por otros investigadores, ya sea con la sacropromontopexia o con la colpopéxia a través de la fijación sacrospinosa, con la ventaja potencial de ser este, un procedimiento de fácil aprendizaje y normatización ${ }^{16}$.

La exposición vaginal de la malla y la erosión visceral son los principales riesgos potenciales del uso de prótesis sintéticas. Si bien no hay evidencia objetiva, se considera que la exposición vaginal y la 
extrusión pueden ser el resultado de la disección vaginal inadecuada, la infección local o atrofia del epitelio, entre otras causas ${ }^{20}$. Se postula, también, que la características biomecánicas de la malla también puede influir en la incidencia de estas complicaciones $^{21}$. A partir de la experiencia con el uso de mallas para la construcción de cabestrillos uretrales, hay consenso de que las mallas sintéticas de polipropileno monofilamento se asocia con menor riesgo de complicaciones en el proceso de integración tisular ${ }^{22}$.

Varios autores ya han demostrado la seguridad y la eficacia de la utilización de mallas de polipropileno monofilamento ${ }^{23,24}$. En nuestra casuística, el uso de mallas de monofilamento con orificios facilitadores de la integración se asoció con una exposición vaginal del (14,7\%) de los casos, los que fueron de fácil resolución, sin secuelas permanentes o impacto sobre el resultado anatómico final, medido a través de POP-Q. Las 5 pacientes en que se detectó la exposición de la malla corresponden a los primeros casos de la serie y se lo relaciona a la curva de aprendizaje inicial. Todas las exposiciones fueron menores a $1 \mathrm{~cm}^{2}$ y se observaron en la línea de sutura, luego se optimizó la forma de colocación, recortando la malla y cubriendo la malla con parte del septo rectovaginal, a nivel del cuerpo perineal reduciéndose así el riesgo.

Los óptimos resultados anatómicos, verificados en el presente estudio, permiten, también, considerar que los agujeros facilitadores de la integración, favorecen la cicatrización del tejido y la resistencia en el interfaz tejido - tela, como ha demostrado experimentalmente (Siniscalhci, en el Congreso CAU 2008). Esto puede representar una ventaja, especialmente en el compartimento vaginal posterior, donde aún no hay consenso sobre el empleo rutinario de prótesis, incluso en los prolapsos acentuados.

Los prolapsos genitales afectan la calidad de vida de las pacientes en la esfera psicológica, social y sexual. El mantenimiento de la función sexual, con la preservación de la longitud y el calibre adecuado para permitir el coito vaginal, es uno de los objetivos de la cirugía pélvica reconstructiva ${ }^{25}$. Existen pocos estudios sobre el impacto del tratamiento del prolapso en la sexualidad de los pacientes ${ }^{26}$, de ahí la importancia de desarrollar herramientas específicas y validadas para ese fin y para su aplicación en la investigación clínica en forma rutinaria relacionada con el empleo de mallas para el tratamiento de los prolapsos, tal como se utiliza en este estudio. En esta serie, 2 pacientes entre las 17 sexualmente activas, $2(11,8 \%)$ desarrollaron dispareunia después del procedimiento, ambas vinculadas a la exposición de la malla, desapareciendo luego de su tratamiento.

En el estudio funcional, el impacto sobre la calidad de vida relacionada con la actividad sexual, medida por ISFI, se observó que hubo ligera mejoría en las sexualmente activas, pero esta diferencia no fue estadísticamente significativa. Por otro lado, en este estudio, los resultados muestran que el uso de mallas en el compartimento vaginal posterior no deterioró la función sexual (Tabla 2).

Tabla 2. Índice de Función Sexual Femenina en pacientes sometidas a colpopexia transcoccígea

\begin{tabular}{|c|c|c|c|c|c|c|c|c|c|}
\hline \multirow[b]{2}{*}{ Dominio IFSF } & \multicolumn{4}{|c|}{ Pre operatorio } & \multicolumn{4}{|c|}{ Pos operatorio } & \multirow[b]{2}{*}{ Valor-p } \\
\hline & $\mathbf{N}$ & Media & Desvio & Mediana & $\mathbf{N}$ & Media & Desvio & Mediana & \\
\hline \multirow[t]{2}{*}{ Deseo } & 1 & 2,6 & 0,5 & 2,4 & 5 & 1,9 & 0,5 & 1,8 & \\
\hline & 7 & & & & & & & & 0,5000 \\
\hline \multirow[t]{2}{*}{ Excitación } & 1 & 3,4 & 0,2 & 3,5 & 5 & 2,6 & 1,2 & 3 & \\
\hline & 7 & & & & & & & & 0,5000 \\
\hline \multirow[t]{2}{*}{ Lubricación } & 1 & 3,5 & 0,4 & 3,6 & 5 & 2,5 & 1,2 & 3 & \\
\hline & 7 & & & & & & & & 0,2500 \\
\hline \multirow[t]{2}{*}{ Orgasmo } & 1 & 3,5 & 0,3 & 3,6 & 5 & 3,0 & 1,0 & 3,6 & \\
\hline & 7 & & & & & & & & 1,0000 \\
\hline \multirow[t]{2}{*}{ Satisfacción } & 1 & 2,8 & 0,7 & 2,8 & 5 & 3,0 & 1,8 & 2 & \\
\hline & 7 & & & & & & & & 1,0000 \\
\hline \multirow[t]{2}{*}{ Dolor } & 1 & 4,5 & 1,6 & 4,8 & 16 & 4,0 & 1,8 & 4,8 & \\
\hline & 7 & & & & & & & & 0,3125 \\
\hline \multirow[t]{2}{*}{ Total } & 1 & 20,3 & 2 & 20,6 & 5 & 17,0 & 6,0 & 19,3 & \\
\hline & 7 & & & & & & & & 0,5000 \\
\hline
\end{tabular}

Test de Wilcoxon para muestras pareadas 


\section{CONCLUSIONES}

La corrección transcoccígea ligamento-específica del prolapso de la pared vaginal posterior con la prótesis NAZCA-R mostró ser segura y efectiva no solo en el aspecto anatómico, según el análisis del POP$\mathrm{Q}$, sino también en la ausencia de complicaciones postoperatorias a largo plazo.

Hubo discreta mejora en la calidad de vida y sexualidad aunque estos resultados no fueron estadísticamente significativos.

\section{REFERENCIAS}

1. Gerten KA, Richter HE. Pelvic floor surgery in the older woman. Clin Obstet Gynecol 2007;50(3):826-843.

2. Hunskaar S, Burgio KL, Clark A et al. Epidemiology of urinary and faecal incontinence and pelvic organ prolapse. In Abrams P, Cardozo L, Khoury S, Wein A (eds): incontinence: 3rd International Consultation 2005;255-312.

3. Olsen AL, Smith VJ, Bergstrom JO, Colling JC, Clark AL. Epidemiology of surgical managed pelvic organ prolapse and incontinence. Obstet Gynecol 1997;89(4):501-506.

4. Capps WF. Rectoplasty and perineoplasty for the symptomatic rectocele: a report of fifty cases. Dis Colon Rectum 1975;18(3): 237-243.

5. De Tayrac R, Picone O, Chauveaud-Lambling A, Fernandez H. A 2-year anatomical and functional assessment of transvaginal rectocele repair using a polypropylene mesh.Int Urogynecol $J$ Pelvic Floor Dysfunct. 2006 Feb;17(2):100-105.

6. Bump RC, Mattiasson A, Bo K, Brubaker LP, DeLancey JOL, Klarskov $\mathrm{P}$ et al. The standardization of terminology of female pelvic organ and pelvic floor dysfunction. Am J Obstet Gynecol 1996;175(1):10-17.

7. Liberman H, Hughes C, Dippolito A. Evaluation and outcome of the delorme procedure in the treatment of rectal outlet obstruction. Dis Colon Rectum 2000;43(2):188-192.

8. Heriot AG, Skull A, Kumar D. Functional and physiological outcome following transanal repair of rectocele. Br J Surg 2004; 91(10):1340-1344.

9. Regadas FS, Regadas SM, Rodrigues LV, Misici R, Silva FR, Regadas Filho FS.. Transanal repair of rectocele and full rectal mucosectomy with one circular stapler: a novel surgical technique. Tech Coloproctol 2005;9(1):63-66.

10. Maher C, Baessler K. Surgical management of posterior vaginal wall prolapse: an evidence-based literature review. Int Urogynecol J Pelvic Floor Dysfunct. 2006;17(1):84-88.

11. Richardson AC. The rectovaginal septum revisited: its relationship to rectocele and its importance in rectocele repair Clin. Obstet. Gynecol 1993;36(4):976-983.

12. Abramov Y, Know C, Gandhi S, Goldberg R, Sand PK. Longterm anatomic outcome of discrete site-specific defect repair versus standard posterior colporrhaphy for the correction of advanced rectocele: 1 year follow-up analysis. Neurourol. Urodyn 2003; 22: 520-521.

13. Burrows LJ, Sewel C, Leffler KS, Cundiff GW. The accuracy of clinical evaluation of posterior vaginal wall defects. Int Urogynecol J Pelvic Floor Dysfunct. 2003;14(3):160-163.
14. De Tayrac R, Picone O, Chauveaud-Lambling A, Fernandez H. A 2-year anatomical and functional assessment of transvaginal rectocele repair using a polypropylene mesh. Int Urogynecol $\mathrm{J}$ Pelvic Floor Dysfunct. 2006 Feb;17(2):100-105.

15. Lim YN, Rane A, Muller R. An ambispective observational study in the safety and efficacy of posterior colporrhaphy with composite Vicryl-Prolene mesh. Int Urogynecol J Pelvic Floor Dysfunct. 2005 Mar-Apr;16(2):126-131.

16. Paraiso MF, Barber MD, Muir TW, Walters MD. Rectocele repair: A randomized trial of three surgical techniques including graft augmentation. Am J Obstet Gynecol. 2006 Dec;195 (6): 1762-1771.

17. Petros PP, Skilling PM. Pelvic floor rehabilitation in the female according to the integral theory of female urinary incontinence. First report. Eur J Obstet Gynecol Reprod Biol. 2001 Feb;94(2): 264-269.

18. Inmon WB. Suspension of the vaginal cuff and posterior repair following vaginal hysterectomy. Am J Obstet Gynecol. 1974; 120(7):977-982.

19. DeLancey JO. Structural anatomy of the posterior pelvic compartment as it relates to rectocele. AAm J Obstet Gynecol. 1999 Apr; 180(4):815-823.

20. Ridgeway B, Chen CC, Paraiso MF. The use of synthetic mesh in pelvic reconstructive surgery. Clin Obstet Gynecol. 2008 Mar;51(1):136-152.

21. Birch C, Fynes NM. The role of synthetic and biological prostheses in the reconstructive pelvic floor surgery. Curr Opin Obstet Gynecol. 2002 Oct; 14(5):527-535.

22. Riccetto C, Miyaoka R, Fraga R, Barbosa R, Dambros M, Teixeira A et al. Impact of the structure of polypropylene meshes in local tissue reaction: in vivo stereological study. Int Urogynecol $\mathrm{J}$ Pelvic Floor Dysfunct. 2008 Aug; 19(8):1117-1123.

23. Cosson M, Debodinance P, Boukerrou M, Chauvet MP, Lobry P, Crépin G et al. Mechanical properties of synthetic implants used in the repair of prolapse and urinary incontinence in women: which is the ideal material?. Int Urogynecol J Pelvic Floor Dysfunct. 2003 Aug; 14(3): 169-178.

24. Maher C, Baessler K, Glazener CM, Adams EJ, Hagen S. Surgical management of pelvic organ prolapse in women, Cochrane Database Syst Rev 3 (2007) CD004014.

25. Weber AM, Walters MD, Pidmonte MR. Sexual function and vaginal anatomy in women before and after surgery for pelvic organ prolapse and urinary incontinence. Am J Obstet Gynecol. 2000 Jun;182(6): 1610-1615.

26. Rogers RG, Kammerer-Doak D, Darrow A, Murray K, Olsen A, Barber M, et al. Sexual function after surgery for stress urinary incontinence and/or pelvic organ prolapse: a multicenter prospective study. Am J Obstet Gynecol. 2004 Jul;191(1):206-210.

Correspondencia autor: Dr. Cássio Riccetto

Servicio de Urología de la Facultad de Ciencias Médicas de la Universidad Estatal de Campinas. Brasil.

E-mail autor: cassio.riccetto@uol.com.br Información artículo: Original - Incontinencia

Trabajo recibido: diciembre 2008

Trabajo aceptado: enero 2008 